\title{
Head-Shaking Nystagmus Depends on Gravity
}

\author{
Antonella Palla, Sarah Marti, and Dominik Straumann \\ Neurology Department, Zurich University Hospital, CH-8091 Zurich, Switzerland
}

Received: 29 December 2003; Accepted: 2 September 2004; Online publication: 2 February 2005

\begin{abstract}
In acute unilateral peripheral vestibular deficit, horizontal spontaneous nystagmus $(\mathrm{SN})$ increases when patients lie on their affected ear. This phenomenon indicates an ipsilesional reduction of otolith function that normally suppresses asymmetric semicircular canal signals. We asked whether head-shaking nystagmus (HSN) in patients with chronic unilateral vestibular deficit following vestibular neuritis is influenced by gravity in the same way as $\mathrm{SN}$ in acute patients. Using a three-dimensional (3-D) turntable, patients $(N=7)$ were placed in different whole-body positions along the roll plane and oscillated $(1 \mathrm{~Hz}$, $\pm 10^{\circ}$ ) about their head-fixed vertical axis. Eye movements were recorded with 3-D magnetic search coils. HSN was modulated by gravity: When patients lay on their affected ear, slow-phase eye velocity significantly increased upon head shaking and consisted of a horizontal drift toward the affected ear (average: $1.2^{\circ} / \mathrm{s}$ $\pm 0.5 \mathrm{SD}$ ), which was added to the gravity-independent and directionally nonspecific SN. In conclusion, HSN in patients with chronic unilateral peripheral vestibular deficit is best elicited when they are lying on their affected ear. This suggests a gravity-dependent mechanism similar to the one observed for SN in acute patients, i.e., an asymmetric suppression of vestibular nystagmus by the unilaterally impaired otolith organs.
\end{abstract}

Keywords: nystagmus, neurootology, vestibular neuritis, otoliths, body tilt

Correspondence to: Antonella Palla $\cdot$ Neurology Department $\cdot$ Zurich University Hospital - CH-8091 Zurich, Switzerland. Telephone: (41) 1-255-5564; fax: (41) 1-255-4507; email: antpalla@access. unizh.ch

\section{INTRODUCTION}

A sudden unilateral peripheral vestibular deficit leads to an asymmetry of tonic vestibular input signals and thus spontaneous eye drift toward the ipsilesional side. Patients avoid lying on their affected ear, because the affected-ear-down position intensifies the vertigo. In fact, an increase of spontaneous nystagmus $(\mathrm{SN})$ is observed in this position, indicating an enhancement of the horizontal semicircular canal imbalance by the changed orientation of the gravity vector relative to the head (Fluur 1973). Such a modulation of canal-mediated SN by gravity strongly suggests an interaction between otolith and semicircular canal pathways.

In the course of vestibular compensation, the velocity of spontaneous eye drift gradually decreases. Patients with chronic unilateral vestibular deficit may yet show some SN in darkness, but not during ocular fixation in the light (Baloh and Honrubia 2001). Nystagmus can still be detected, however, after shaking the head rapidly over 20 to 30 cycles in the horizontal plane (Kamei et al. 1964) or after wholebody oscillation on a turntable about an Earthvertical axis (Fetter et al. 1990; Katsarkas et al. 2000). This so-called head-shaking nystagmus (HSN) is considered to be a sensitive symptom for detecting asymmetries in the vestibular system (Hain et al. 1987; Fetter et al. 1990; Hain and Spindler 1993).

The presence of HSN reflects a directional imbalance of the vestibuloocular reflex (VOR) in the highfrequency range after unilateral vestibular lesions. During high-acceleration head rotations, the VOR is mainly generated by the excited side (Ewald 1892), because the nonlinear pathway of the inhibited side is driven into inhibitory cutoff (Lasker et al. 2000). For nystagmus to appear after head oscillation has 
stopped, the directionally asymmetric response must have been stored during head shaking to be discharged thereafter. Therefore to elicit HSN, the brainstem network that transiently accumulates the vestibular velocity signals, the so-called "velocity storage" (Raphan et al. 1979), must be operative to receive and perpetuate the input that predominantly comes from the healthy ear (Fetter et al. 1990; Hain and Zee 1992). Consequently, HSN generally beats toward the healthy ear. Central mechanisms under the control of the cerebellum, however, may lead to a secondary phase of nystagmus in the opposite direction or even a reversed nystagmus right from the beginning (Asawavichiangianda et al. 1999).

We asked whether the primary phase of horizontal HSN (HSNh) in patients with chronic unilateral vestibular deficit after vestibular neuritis is influenced by the orientation of the gravity vector and whether such a gravity-dependent modulation would resemble the one seen in horizontal SN (SNh) of patients with acute unilateral vestibular deficit. This would indicate that otolith-mediated mechanisms interfering with the asymmetry of semicircular canal signals are the same for both SNh and HSNh.

\section{METHODS}

\section{Definition}

The term "head-shaking nystagmus" (HSN) was applied to describe nystagmus appearing after oscillation of the head in space. This broader definition of HSN, which goes beyond head shaking on the trunk at the bedside, is in line with the existing literature and includes whole-body oscillation on a turntable (Fetter et al. 1990; Katsarkas et al. 2000).

\section{Subjects}

Three-dimensional (horizontal, vertical, torsional) eye movements before, during, and after head shaking were recorded in seven patients (four male, 28-77 years) with chronic unilateral peripheral vestibular deficit after vestibular neuritis (Schuknecht and Kitamura 1981; Arbusow et al. 2000). Another four patients also participated in the study, but opted not to complete the experimental protocol because of nausea. The diagnosis was based on the patient's history and bedside testing. The clinical examination was performed by an experienced neurootologist (D.S.). Clinically, horizontal head-shaking nystagmus under Frenzel glasses was present in all seven patients. Quantitative head impulse testing with search coils confirmed the unilateral peripheral vestibular deficit (Aw et al. 1996). In six patients the deficit was right- sided, in one patient left-sided. Because search-coil head impulse testing is more sensitive than caloric testing in chronic patients after vestibular neuritis (Schmid-Priscoveanu et al. 2001), caloric irrigation was not performed in all patients. The average duration since the onset of the vestibular deficit was 3.5 years (range: 3 months-10 years). The comparison group consisted of 12 healthy subjects (six male, 25-59 years).

The subjects gave their consent to participate in this study after being informed of the experimental procedures. The protocol was approved by a local ethics committee and was in accordance with the ethical standards laid down in the Declaration of Helsinki for research involving human subjects.

\section{Experimental set-up}

Subjects were seated upright on a turntable with three servo-controlled motor-driven axes (prototype built by Acutronic, Jona, Switzerland). The head was restrained with an individually molded thermoplastic mask (Sinmed BV, Reeuwijk, The Netherlands). Subjects were positioned so that the center of the interaural line was at the intersection of the three axes of the turntable. Pillows and safety belts minimized movements of the body. The chair was pitched $20^{\circ}$ in the nose-down direction to position the lateral semicircular canals approximately horizontal.

\section{Eye and head movement recording}

Three-dimensional (3-D) eye and head movements were recorded with dual search coils (Skalar Instruments, Delft, The Netherlands). The coil frame (side length: $0.5 \mathrm{~m}$ ) generated three orthogonal digitally synchronized magnetic wave field signals of 80,96 , and $120 \mathrm{kHz}$. A digital signal processor computed a fast Fourier transform in real-time on the digitized search coil signal to determine the voltage induced on the coil by each magnetic field (system by Primelec, Regensdorf, Switzerland). Coil orientation could be determined with an error of less than $7 \%$ over a range of $\pm 30^{\circ}$ and with a noise level of less than $0.05^{\circ}$ (root mean squared deviation).

Search coil annuli were calibrated with a method described elsewhere (Straumann et al. 1995). A dual search coil was placed around the cornea of the right eye after local anesthesia with oxybuprocaine $0.4 \%$. A second coil for measuring head movements was fixed on the front teeth via silicon dental impression paste (Blu-Mousse ${ }^{\circledR}$ Classic, Parkell, Inc., Farmingdale, NY, USA). Eye, head, and chair position signals were digitized at $1000 \mathrm{~Hz} /$ channel with 16-bit resolution, 
and stored on a computer hard disk for offline processing.

\section{Experimental protocol}

A chair-fixed laser dot was projected straight ahead onto a tangent screen at a distance of $0.59 \mathrm{~m}$ in front of the subject's eyes. Every $2 \mathrm{~s}$ the laser dot was turned on for a duration of $20 \mathrm{~ms}$. Subjects were instructed to look at the laser dot and to keep their eyes at this position during the off periods. The short duration of on periods ensured that the smooth pursuit system was not activated.

The chair was rotated in the roll plane in $45^{\circ}$ steps from the left ear-down to the right ear-down position (five positions: $90^{\circ}$ and $45^{\circ}$ left ear-down; upright; $45^{\circ}$ and $90^{\circ}$ right ear-down). Each position was held for a $90 \mathrm{~s}$ period consisting of three phases: (1) $30 \mathrm{~s}$ of stationary chair position; (2) $30 \mathrm{~s}$ of turntable oscillation about the head-fixed vertical axis (approximately orthogonal to the lateral semicircular canals; see Experimental set-up) with an amplitude of $10^{\circ}$ and a frequency of $1 \mathrm{~Hz}$; (3) $30 \mathrm{~s}$ of stationary chair position. Eye movements during the first interval corresponded to spontaneous nystagmus (SN), during the second interval to the vestibuloocular reflex (VOR), and during the third interval to head-shaking nystagmus (HSN). Note that oscillating the subject about the head-fixed yaw axis implied some degree of perrotatory VOR dumping, except in the upright position, in which the rotation axis was Earth-vertical (Bockisch et al. 2003).

\section{Data analysis}

Search coil signals from the right eye and the head were processed with interactive programs written in MATLAB $^{\text {TM }}$ Version 6. 3-D positions of eye and head were expressed as rotation vectors (Haustein 1989), and corresponding 3-D velocities as angular velocity vectors (Hepp 1990). The transverse plane of the head-fixed coordinate system was aligned with the Earth-horizontal with the head pitched $20^{\circ}$ nosedown. In this position, defined as upright, the horizontal semicircular canals were approximately parallel to the Earth-horizontal plane. For convenience, the lengths of rotation vectors and angular velocity vectors were given in degrees and degrees per second $(\% / s)$, respectively. According to the right-hand rule, eye rotations to the left, down, and clockwise from the subject's point of view are positive. To facilitate the analysis, 3-D eye movement directions in the patient with the left-sided vestibular lesion were mirrored, as if the right ear had been affected by the vestibular neuritis. This was accomplished by multiplying the horizontal and torsional eye and head movement components by $(-1)$. Thus in the analysis of this patient's data, it was as if the right side was the affected side.

In each turntable position, eye movements during intervals $10 \mathrm{~s}$ before and $10 \mathrm{~s}$ after head shaking were analyzed by interactively selecting sections of slowphase eye movements between quick phases of nystagmus. To determine the dynamics of the ocular response during whole-body oscillation, eye velocity was desaccaded by overlaying all cycles and comput-
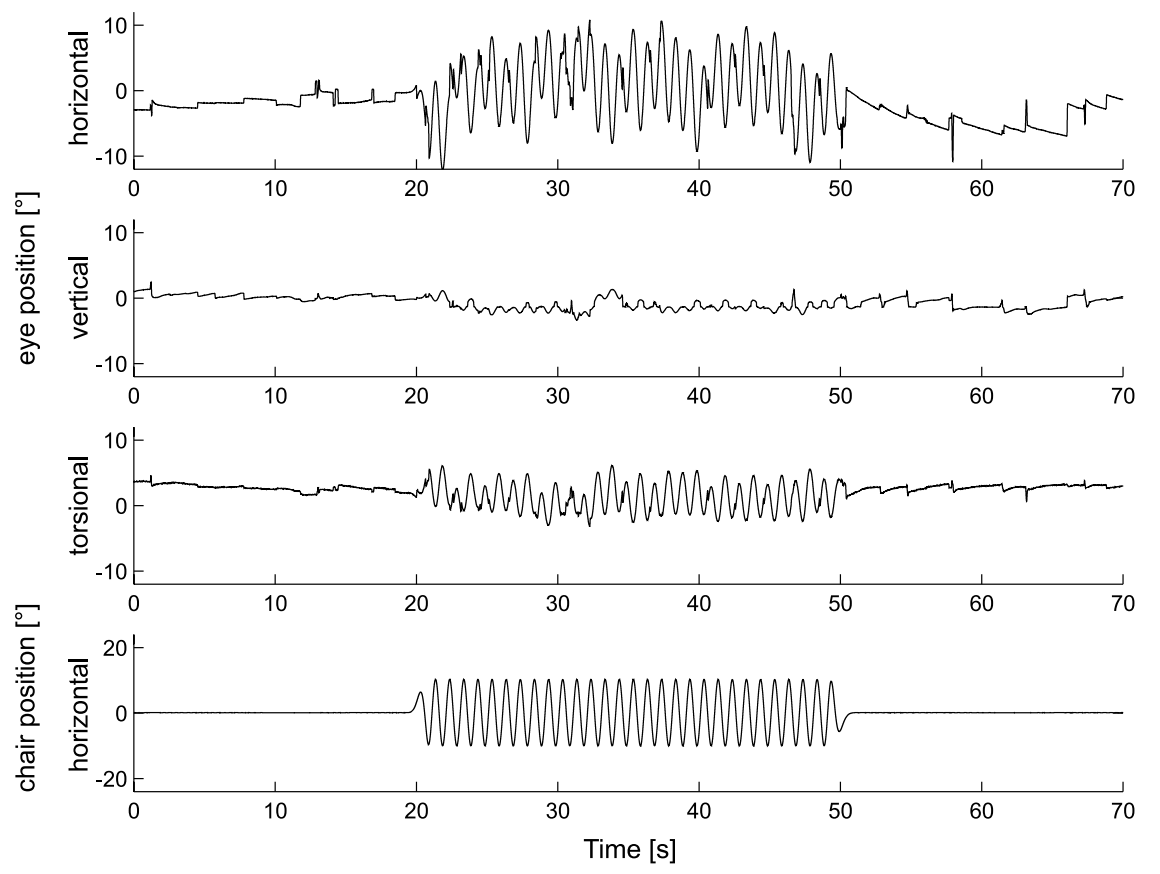

FIG. 1. Three-dimensional position traces (upper three panels: horizontal, vertical, torsional) of the right eye in an upright sitting patient (P.I.) with a right-sided peripheral vestibular deficit. Eye movements to the left, down, and clockwise, as seen by the subject, are positive according to the right-hand rule. Chair position (lowest panel) oscillated in the horizontal direction only $\left(1 \mathrm{~Hz}, \pm 10^{\circ}\right)$. Traces are clipped $20 \mathrm{~s}$ before and $20 \mathrm{~s}$ after the turntable oscillation. 

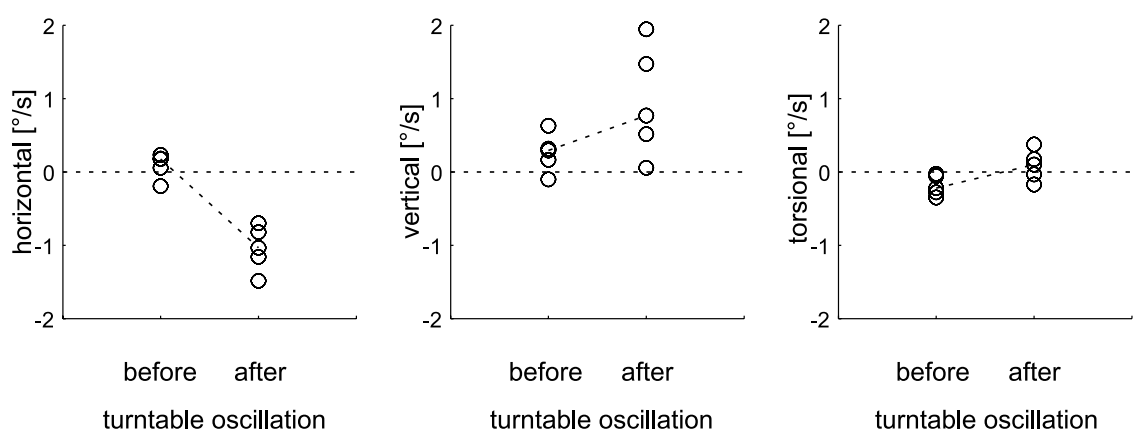

FIG. 2. Average velocities of individual slow phases (open circles) of nystagmus before and after horizontal turntable oscillation in the same upright sitting patient as in Figure 1. Eye movement directions according to the right-hand rule. Selected slow phases were within $10 \mathrm{~s}$ before and after turntable oscillation. Dashed lines connect averages of data points before and after turntable oscillation. ing the median eye response (Schmid-Priscoveanu et al. 2000). Then, horizontal eye velocity was plotted against horizontal chair velocity. The slope of the first-linear regression yielded the gain, its offset the velocity bias.

\section{RESULTS}

Figure 1 shows three-dimensional (3-D) eye position traces measured in an upright-positioned patient with a right-sided peripheral vestibular deficit. Before turntable oscillation, only a small drift was noted. After oscillation, horizontal drift, directed toward the affected right side, increased considerably more than vertical and torsional drifts. Hence the resulting "head-shaking nystagmus" was mainly horizontal.

For the same example, average velocities of nystagmus slow phases before and after turntable oscillation are depicted in Figure 2. The largest change of slow-phase eye velocity (averages connected by dashed lines) occurred in the horizontal eye movement plane: before head shaking, velocities scattered around zero; after head shaking, the eyes drifted toward the right side, i.e., the side of the vestibular lesion (unpaired t-test: $p<0.01)$. Downward-directed vertical drift increased slightly after head shaking, but data points scattered widely $(p=0.09)$. Torsional drift was directed counterclockwise before head shaking, but reversed its direction after head shaking $(p=0.04)$.

Figure 3 shows average horizontal eye velocities before (circles) and after head shaking (stars) in different whole-body positions along the roll plane in a healthy subject (Fig. 3A) and in the same patient as in the previous example (Fig. 3B). In the healthy subject, the horizontal velocity of spontaneous drift and drift after head shaking scattered around zero independent of body position. In the patient, the horizontal spontaneous drift did not modulate with gravity. Horizontal drift velocity after head shaking, however, showed a clear gravity-dependent gradient with an increasing rightward drift from $90^{\circ}$ left ear-down to $90^{\circ}$ right ear-down. Accordingly, slow-phase eye velocity toward the affected right side was most prominent when the patient was lying on the affected right ear.

Figure 4 depicts slow-phase eye velocity of all patients in left ear-down, upright, and right ear-down whole-body positions before and after head shaking. For comparison, the differences of drift velocities between the same conditions in healthy subjects are also plotted. Recall that in all patients the right side corresponds to the affected side, i.e., eye movement

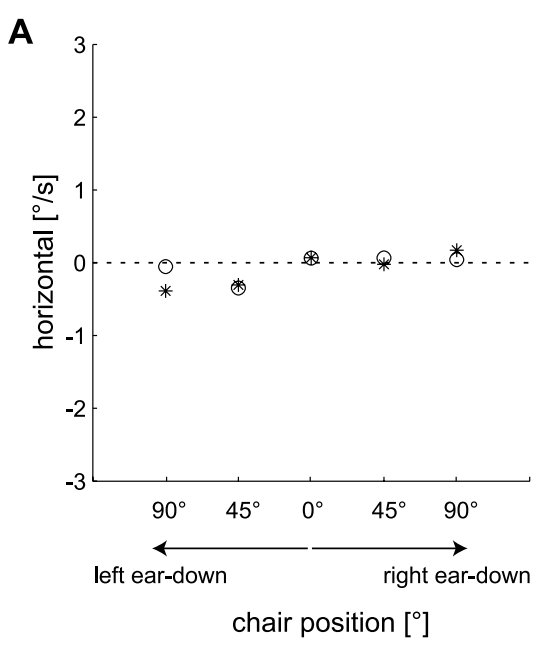

chair position $\left[^{\circ}\right]$

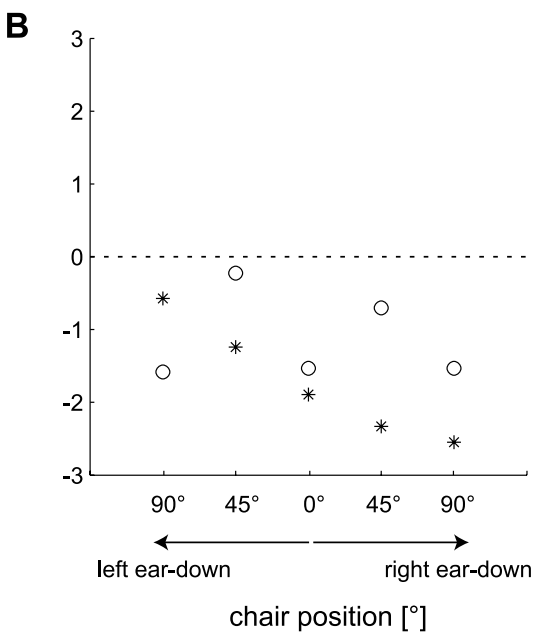

FIG. 3. Examples of average horizontal slow-phase eye velocities before (circles) and after (stars) horizontal turntable oscillation in different body positions along the roll plane. Eye velocity to the left is positive. A Healthy subject (A.P.). B Same patient as in Figures 1 and 2. Note the increase of horizontal eye velocity toward the affected right side after oscillation when the patient is rolled toward the affected right ear. 
data in the only one patient with a left-sided lesion were mirrored (see Methods). Horizontal slow-phase eye velocity in the upright and nonaffected (=left) eardown positions was not significantly different from zero before (open circles; triangle $\equiv$ mirrored data point) and after (stars; triangle $\equiv$ mirrored data point) head shaking (ANOVA: $\left.p_{\text {horizontal }}=0.94\right)$. In the affected (= right) ear-down position, however, head shaking elicited a significant (paired $t$-test: $p<0.01$ ) change of horizontal slow-phase eye velocity toward the undermost ear (average increase of slow-phase eye velocity by head shaking: $\left.1.2^{\circ} / \mathrm{s} \pm 0.5 \mathrm{SD}\right)$. There was no
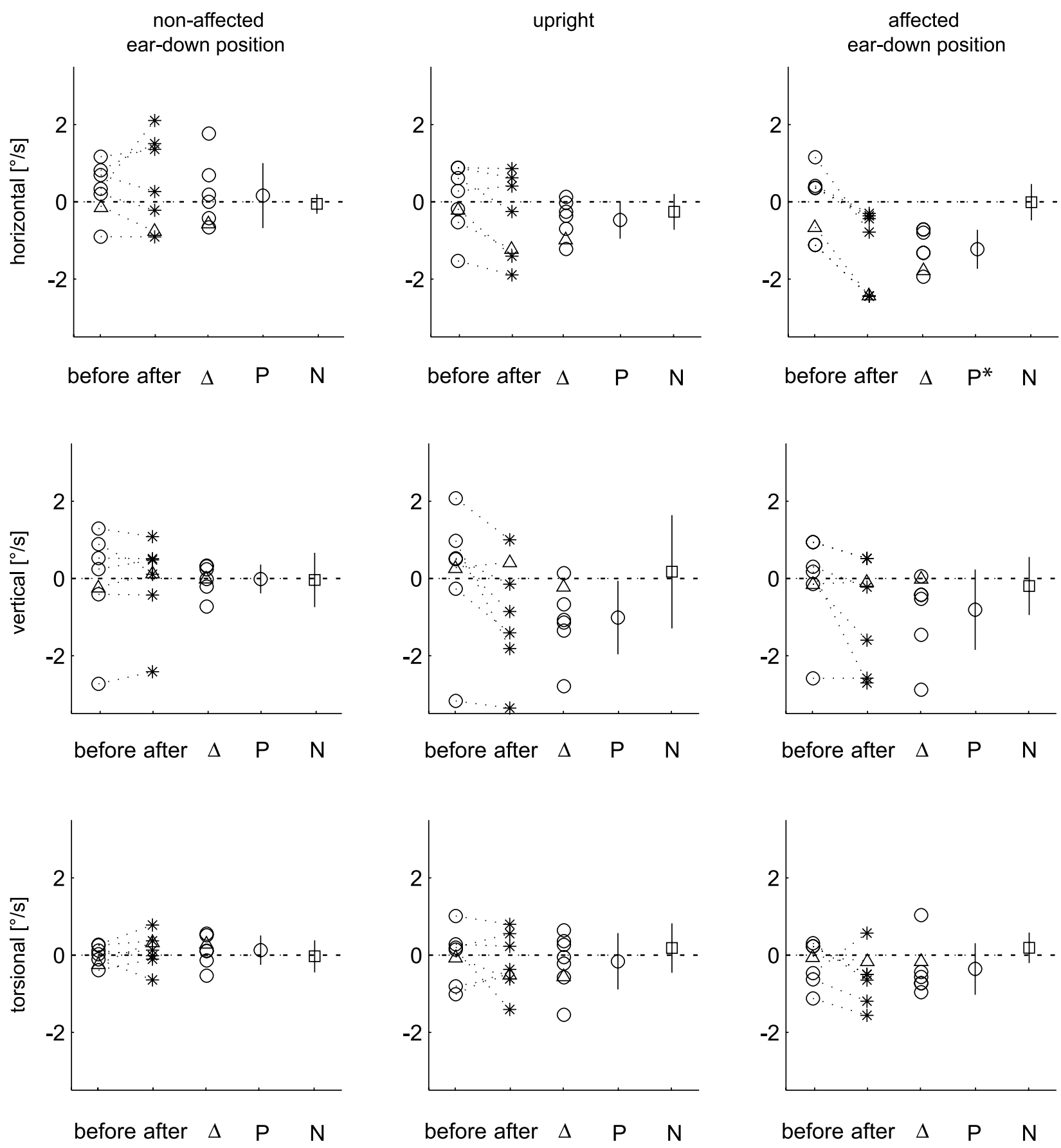

FIG. 4. Comparisons between average slow-phase eye velocities before (open circles) and after (stars) horizontal turntable oscillation in all patients in the non-affected ear-down, upright, and affected ear-down positions. Eye movement directions according to righthand rule. In the ear-down positions, each data point on one side corresponds to the average eye velocity measured in the $90^{\circ}$ and $45^{\circ}$ body positions. In the one patient with a left-sided deficit (data depicted as triangles), eye movement directions were mirrored, thus, for analysis, the right ear was always the affected ear. Dotted lines connect eye velocities of individual patients. $\Delta$ : Difference between slow-phase eye velocities before and after oscillation. P: Average $\Delta$ $( \pm 1 \mathrm{SD})$ in patients. $\mathrm{N}$ : Average $\Delta( \pm 1 \mathrm{SD})$ in healthy subjects. 
A

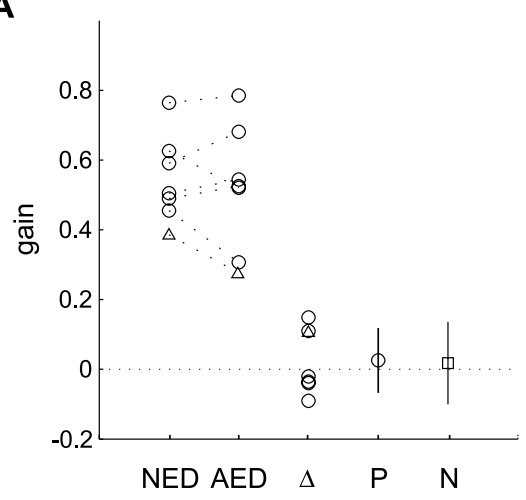

B

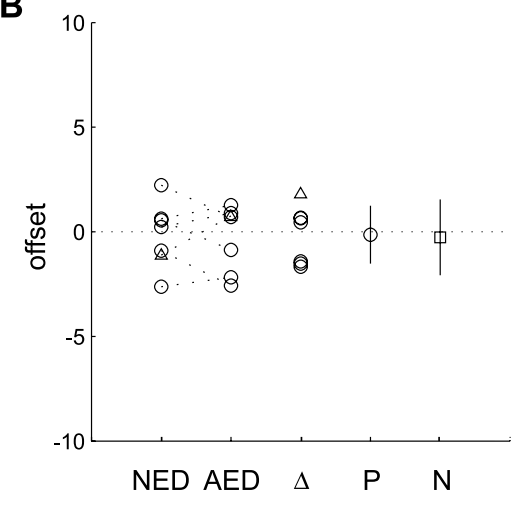

FIG. 5. Parameters of sine fits through desaccaded horizontal slow-phase velocity during horizontal turntable oscillation. Comparison between averages of individual patients (open circles; triangle for mirrored data in the one patient with a left-sided deficit) in affected ear-down (AED) and non-affected ear-down (NED) roll body positions. $\Delta$ : Differences, $\mathrm{P}$ : Average $\Delta( \pm 1 \mathrm{SD}), \mathrm{N}$ : Average $\Delta( \pm 1 \mathrm{SD})$ in healthy subjects. A Gain. B Offset. significant effect of horizontal head shaking on slowphase velocity in the vertical and torsional directions (ANOVA: $\left.p_{\text {torsional }}=0.76 ; p_{\text {vertical }}=0.79\right)$. In the healthy subjects, no significant differences between drift velocities before and after head shaking (open squares with error bars) were observed in any roll body position tested.

We asked whether the influence of gravity on ocular responses was not only apparent after, but also during head shaking. Figure 5A shows average gain values of the horizontal vestibuloocular reflex elicited by horizontal turntable oscillation in the non-affected ear-down and the affected ear-down positions. In all patients, no significant differences were noted between the gains in these roll body positions. As depicted in Figure 5B, the average offset of the fitted sine to horizontal ocular velocity during horizontal turntable oscillation was not different from zero in both side positions.

\section{DISCUSSION}

This study analyzed the influence of gravity on threedimensional (horizontal, vertical, and torsional) slowphase eye velocity before and after horizontal head shaking in patients with chronic unilateral peripheral vestibular deficit after vestibular neuritis. Head shaking was applied by whole-body oscillation about a head-vertical axis that was oriented approximately perpendicular to the lateral semicircular canals. We emphasize that this type of head shaking does not exactly represent the head shaking used at the bedside. However, the aim of our study was to apply the head shaking stimulus in a reproducible way in different head roll orientations, which could not be carried out if the head oscillation were performed by hand.

In all roll positions, including upright, patients showed horizontal eye drift both before (=horizontal spontaneous nystagmus, $\mathrm{SNh}$ ) and after (=horizontal head-shaking nystagmus, HSNh) head shaking. Slowphase eye velocity of SNh scattered around zero and the direction of nystagmus was independent of the side of the vestibular deficit. In fact, average slowphase eye velocity among the seven patients was not influenced by gravity (=no positional nystagmus) and was not significantly different from zero in all body positions, which was most likely the result of vestibular compensation.

When horizontal head shaking was applied to patients in the upright position, slow-phase eye velocity did not significantly change from $\mathrm{SNh}$. This result is in agreement with the finding of Katsarkas et al. (2000) that, in patients with unilateral peripheral vestibular loss, no consistent velocity bias toward the lesioned side for peak velocities of oscillatory head shaking $<160^{\circ} / \mathrm{s}$ was elicited. However, when head shaking was applied to patients lying on their affected ear, we found a significant horizontal velocity bias toward this side. Together with the observation of unaffected horizontal slow-phase eye velocity by head shaking when patients were lying on their healthy ear, we can conclude that the head tilt toward the affected side had a facilitating effect (Fig. 4). Because of the dynamic restrictions of our turntable, the peak velocity was lower than with manual head shaking. This probably explains why turntable oscillation did not elicit HSNh in the majority of uprightseated patients, even when HSNh in the same patients was visible under the Frenzel glasses after manual head shaking in upright position. In addition, we cannot exclude that this difference between turntable and manual head shaking might be attributable to the lack of proprioceptive neck signals during the whole-body oscillation. In fact, the cervicoocular reflex is known to increase its gain in patients with vestibular deficits (Heimbrand et al. 1996). Nevertheless, our study demonstrates that a significant portion of head-shaking nystagmus depends on vestibular stimulation.

The gravity dependence of HSNh in our chronic patients is comparable to the gravity dependence of 
$\mathrm{SNh}$ in acute patients, in whom horizontal slow-phase eye velocity increases when they lie on the affected ear. This indicates an interaction between otolith and semicircular canal signals. The exact nature of this interaction is still uncertain. Fluur's hypothesis on the gravity dependence of $\mathrm{SNh}$ in acute patients is based on the presumption that otolith signals are used by the central nervous system to reduce the imbalance between semicircular canal signals (Fluur 1973). Because otolith signals are directionally polarized, i.e., mainly responding to ipsilateral head roll (Fernandez and Goldberg 1976), such an otolithmediated suppression of vestibular nystagmus is weaker when the head is rolled toward the affected ear, provided the otolith organs or otolith afferents are impaired on this side as well. It is indeed likely that the utricle has been at least partially afflicted in our patients, because of the predominant involvement of the superior division of the vestibular nerve in vestibular neuritis (Fetter and Dichgans 1996). As slow-phase eye velocities of both SNh and HSNh increase when patients are positioned with the affected ear down, we conjecture that the mechanisms of gravity dependence for $\mathrm{SNh}$ and $\mathrm{HSNh}$ are similar, i.e., the otolith-mediated suppression of asymmetric tonic semicircular canal signals is more effective when patients are lying on the ear that provides normal otolith signals to the central nervous system.

We can only speculate at what level the modulation of HSNh takes place. Because the gain of the vestibuloocular reflex during head shaking did not differ between different body positions along the roll plane, the gravity dependence of HSNh cannot be explained by a head-position-dependent variation of vestibular input into velocity storage. Rather, otolith signals seem to influence the velocity storage mechanism itself, most likely by shortening the time constant (=dumping mechanism) via the vestibulocerebellum, specifically the nodulus (Hain et al. 1988). In fact, our results suggest that the gravity dependence of HSNh, and perhaps also of SNh, represent an asymmetry of the cerebellar "dumping" mechanism as a result of a unilateral otolith deficit.

An alternative explanation of $\mathrm{SNh}$ and $\mathrm{HSNh}$ is based on the gravito-inertial force (GIF) resolution hypothesis by Merfeld and colleagues on how the brain solves the tilt-translation dilemma (Merfeld et al. 1993; Merfeld and Young 1995). These authors propose an internal representation of gravity by the central nervous system. Any difference occurring between the GIF vector sensed by the otoliths and the internal gravity vector is interpreted as a vector of linear translation. Extending the GIF resolution hypothesis to patients with unilateral peripheral vestibular hypofunction, the observed gravity dependence of SNh and HSNh can be predicted as follows:
If patients receive less otolith input when lying on the affected ear, the difference between the GIF vector and the internal vector (i.e., the estimated headhorizontal translation in the direction of the uppermost ear) is larger, which leads to an increase of the horizontal slow-phase eye velocity ( $\mathrm{SNh}$ in acute patients, HSNh in chronic patients) toward the undermost ear.

As suggested by earlier experiments on static and dynamic otolith-ocular reflexes in the roll plane in the presence of unilateral peripheral vestibular hypofunction, there is a central compensation of the unilateral utricular deficit (Schmid-Priscoveanu et al. 1999). This would explain our finding that, in the chronic state of a unilateral vestibular deficit, $\mathrm{SNh}$ was not significantly different between the two ear-down positions. However, the gravity dependence of HSNh in our chronic patients clearly indicates that the central compensation of the utricular asymmetry is incomplete, i.e., can be unmasked by dynamic semicircular canal stimulation, if the canal signals are asymmetrically impaired.

In conclusion, we have shown a clear modulation of HSNh by gravity in patients with chronic unilateral peripheral vestibular deficit due to vestibular neuritis. The increase in horizontal slow-phase velocity of HSNh, which appeared when patients were lying on the affected ear, was the result of a head-shakinginduced drift added to SNh. Thus the most efficient body position to elicit HSNh, and therefore to unmask the unilateral involvement of otolith organs or their afferents, is the roll position with the affected ear down. In this position HSNh may be present, even if central compensation has already abolished the gravity dependence of $\mathrm{SNh}$.

\section{ACKNOWLEDGMENTS}

We thank Prof. S. Palla and Dr. M. Bertschinger for technical advice; S. Bizzarri, T. Schmüickle, and A. Züger for technical assistance. This work was supported by Swiss National Science Foundation (3231-051938.97/3163465.00), and Betty and David Koetser Foundation for Brain Research (Zurich, Switzerland).

\section{REFERENCES}

Arbusow V, Strupp M, Wasicky R, Horn AK, Schulz P, Brandt T. Detection of herpes simplex virus type 1 in human vestibular nuclei. Neurology 55:880-882, 2000.

Asawavichiangianda S, Fujimoto M, Mai M, Desroches H, Rutka J. Significance of head-shaking nystagmus in the evaluation of the dizzy patient. Acta Otolaryngol. Suppl. 540:27-33, 1999.

Aw ST, Halmagy GM, Haslwanter T, Curthoys IS, Yavor RA, Todd MJ. Three-dimensional vector analysis of the human vestibu- 
loocular reflex in response to high-acceleration head rotations. II. Responses in subjects with unilateral vestibular loss and selective semicircular canal occlusion. J. Neurophysiol. 76:40214030, 1995.

Baloh RW, Honrubia V. Clinical Neurophysiology of the Vestibular System, 3rd ed., Oxford University Press, New York, 2001.

Bockisch CJ, Straumann D, Haslwanter T. Eye movements during multi-axis whole-body rotations. J. Neurophysiol. 89:355-366, 2003.

EwaLd EJ. Physiologische Untersuchungen über das Endorgan des Nervus Octavus. Bergmann, Wiesbaden, Germany, 1892.

Fernandez C, Goldberg JM. Physiology of peripheral neurons innervating otolith organs of the squirrel monkey. I. Response to static tilts and to long-duration centrifugal force. J. Neurophysiol. 39:970-984, 1976.

FetTer M, Dichgans J. Vestibular neuritis spares the inferior division of the vestibular nerve. Brain 119:755-763, 1996.

Fetter M, Zee DS, Koenig E, Dichgans J. Head-shaking nystagmus during vestibular compensation in humans and rhesus monkeys. Acta Otolaryngol. 110:175-181, 1990.

FLUUR E. Interaction between the utricles and the horizontal semicircular canals. IV. Tilting of human patients with acute unilateral vestibular neuritis. Acta Otolaryngol. 76:349-352, 1973.

HAIN TC, SPIndLer J. Head-shaking nystagmus. In: Sharde JA, Barbo HO (Eds) The Vestibulo-Ocular Reflex and Vertigo. Raven Press, New York, 1993.

Hain TC, ZeE DS. Velocity storage in labyrinthine disorders. Ann. N. Y. Acad. Sci. 656:297-304, 1992.

Hain TC, FetTer M, Zee DS. Head-shaking nystagmus in patients with unilateral peripheral vestibular lesions. Am. J. Otolaryngol. 8:36-47, 1987.

Hain TC, Zee DS, Maria BL. Tilt suppression of vestibulo-ocular reflex in patients with cerebellar lesions. Acta Otolaryngol. 105:13-20, 1988.

HAUSTEIN W. Considerations on Listing's law and the primary position by means of a matrix description of eye position control. Biol. Cybern. 60:411-420, 1989.

Heimbrand S, Bronstein AM, Gresty MA, Faldon ME. Optically induced plasticity of the cervico-ocular reflex in patients with bilateral absence of vestibular function. Exp. Brain Res. 112:372-380, 1996.

Hepp K. On Listing's law. Commun. Math. Phys. 132:285-292, 1990.

Kamei T, Kimura K, Kaneko H. Re-evaluation of the head shaking test as a method of nystagmus provocation. Jpn. J. Otol. 67:1530-1534, 1964

Katsarkas A, Smith H, Galiana H. Head-shaking nystagmus (HSN): the theoretical explanation and the experimental proof. Acta Otolaryngol. 120:177-181, 2000.

Lasker DM, Hullar TE, Minor LB. Horizontal vestibuloocular reflex evoked by high-acceleration rotations in the squirrel monkey. III. Responses after labyrinthectomy. J. Neurophysiol. 83:2482-2496, 2000.

Merfeld DM, Young LR. The vestibulo-ocular reflex of the squirrel monkey during eccentric rotation and roll tilt. Exp. Brain Res. 106:111-122, 1995

Merfeld DM, Young LR, Oman CM, Shelhamer MJ. A multidimensional model of the effect of gravity on the spatial orientation of the monkey. J. Vestib. Res. 3:141-161, 1993.

Raphan T, Matsuo V, Cohen B. Velocity storage in the vestibuloocular reflex arc (VOR). Exp. Brain Res. 35:229-248, 1979.

Schmid-Priscoveanu A, Straumann D, Böhmer A, Obzina H. Vestibulo-ocular responses during static head roll and three-dimensional head impulses after vestibular neuritis. Acta Otolaryngol. 119:750-757, 1999.

Schmid-Priscoveanu A, Straumann D, Kori AA. Torsional vestibuloocular reflex during whole-body oscillation in the upright and the supine position. I. Responses in healthy human subjects. Exp. Brain Res. 134:212-219, 2000.

Schmid-Priscoveanu A, Böhmer A, Obzina H, Straumann D. Caloric and search-coil head-impulse testing in patients after vestibular neuritis. J. Assoc. Res. Otolaryngol. 2:72-78, 2001.

Schuknecht HF, Kitamura K. Second Louis H. Clerf lecture. Vestibular neuritis. Ann. Otol. Rhinol. Laryngol. Suppl. 90:1-19, 1981.

Straumann D, Zee DS, Solomon D, Lasker AG, Roberts D. Transient torsion during and after saccades. Vis. Res. 35:3321-3334, 1995. 